\title{
Acarbose promotes remission of both early and late dumping syndromes in post-bariatric patients
}

This article was published in the following Dove Press journal:

Diabetes, Metabolic Syndrome and Obesity:Targets and Therapy

7 December 2016

Number of times this article has been viewed

\author{
Flavio A Cadegiani' \\ Osvalmir Sá Silva² \\ 'Division of Endocrinology and \\ Metabolism, Department of Medicine, \\ Escola Paulista de Medicina, \\ Universidade Federal de São Paulo, \\ São Paulo, SP, ${ }^{2}$ Corpometria Institute, \\ an Obesity and Endocrinology Center, \\ Brasilia, DF, Brazil
}

Objective: Acarbose is a glucosidase inhibitor that slows carbohydrate digestion. It could thus be effective to promote remission of dumping syndrome (DS). Previous studies associating acarbose and late dumping, although not early dumping, have been reported. Herein, we aimed to evaluate the role of acarbose in dumping syndrome prevention and treatment and in resistive exercises resistance in bariatric subjects.

Methods: Bariatric patients with DS and complete adherence to diet plan and resistive exercises were included ( $\mathrm{n}=25)$. Number of early and late episodes, self-referred intensity of each episode, and ability to increase intensity of resistive exercise were evaluated, on a $0-10$ scale. Acarbose was administered orally ( $50 \mathrm{mg}$ ) for 6 months, 4-5 times a day before meals.

Results: Acarbose administration was associated with a decrease in the number of early (2.18-0.31) and late (2.79-0.12) episodes per week and intensity of each episode (6.10-1.65) and an increase in the ability to perform resistive exercises (3.03-7.12). Complete remission of DS was seen in 21 patients (84\%), which persisted for 6 months with the use of acarbose.

Conclusion: Acarbose prevented dumping in almost all studied subjects and helped improve exercise capacity.

Keywords: dumping syndrome, bariatric surgery, post-bariatric, obesity

\section{Introduction}

Dumping syndrome (DS) is the effect of rapid gastric emptying with consequent hyperosmolar jejunal chime, inappropriate gut-hormone release, and rapid glucose absorption, and it is particularly common among post-bariatric surgery patients, as it occurs in up to $75 \%$ of patients after Roux-en-Y gastric bypass surgery. ${ }^{1-5}$ DS can be either early - when symptoms happen between 30 and 60 min as a result of rapid instillation of meals into the small bowel and decreased blood volume due to intraluminal fluid sequestration, and most symptoms are abdominal (bloating and abdominal pain) and systemic (palpitations, fatigue, tachycardia, lightheadedness, and syncope) - or late, which is thought to be caused by hyperinsulinemic hypoglycemia, and the most commonly observed symptoms and signs are due to neuroglycopenia and hyperadrenergic state (decreased consciousness, shakiness, and difficulty to concentrate). It is important to note that often patients may present both early and late DS at the same meal. DS reduces the quality of life as symptoms are usually severe and can limit sports capacity and everyday activities..$^{1-7}$

Current approaches for DS treatment comprise dietary recommendations ${ }^{3,4,6}$ such as small and frequent meals, inclusion of fiber and protein in every meal, and ingestion
Correspondence: Flavio A Cadegian Division of Endocrinology and Metabolism, Department of Medicine, Escola Paulista de Medicina, Universidade Federal de São Paulo, R. Pedro de Toledo 78I, 04039-032 São Paulo, SP, Brazil Tel +5561981395395

Fax +55 6I 33464733

Email flaviounb@gmail.com 
of guar gum and pectin. Pharmacological options for DS are limited and mostly symptomatic, such as tincture opium for diarrhea, ${ }^{7}$ meclizine, promethazine, and proton pump inhibitors. ${ }^{3,6}$ Octreotide has been shown to be markedly effective to improve the quality of life in DS, ${ }^{8,9}$ despite commercial and financial limitations and significant side effects. However, most of the potential benefits of the current approaches are observed only in late DS, but not in the early DS, nor in exercise-induced dumping, a condition described that affects some post-bariatric athletes, ${ }^{10-12}$ specially during intense physical activities, ${ }^{10,11}$ whose pathophysiology remains uncertain but may be due to exacerbation of gastrointestinal physiological adaptions to physical activity. ${ }^{11}$

Indeed, there is a lack of previous papers focusing on the management of exercise-induced DS, except for expert opinion and experience and nonscientific recommendations. ${ }^{13}$ A recent review on management of DS has been published and reinforces the lack of papers about correlations between physical activity and DS. ${ }^{4}$

Acarbose, a glucosidase inhibitor that slows carbohydrate digestion and is primarily prescribed as an antidiabetic agent has been shown to be effective in late DS, ${ }^{6,14-16}$ but has not been studied for early DS; rationale of protection of late dumping by acarbose is based on the fact that this drug delays glucose absorption; thus, hyperinsulinemic hypoglycemia due to excessive insulin release by incretin and by direct glucose stimulation is prevented by acarbose. ${ }^{14,16}$ Furthermore, acarbose has the potential benefit to prevent beta-cell hypertrophy and hyperplasia; indeed, hyperinsulinemic hypoglycemia, one of the main cornerstone aspects of DS, has a significant improvement by acarbose. ${ }^{6,17,18}$

Other studies with acarbose in DS have not been performed, as severe side effects such as excessive flatulence ${ }^{14}$ had limited its use. Despite the benefits, acarbose has not been standardized as part of protocols to manage DS symptoms. $^{3}$

Despite the symptomatic limitations of acarbose, the lack of efficient options to prevent and improve DS and the strong likelihood of acarbose to improve both early and late DS encouraged us to undertake this study. Therefore, we aimed to evaluate the role of acarbose in prevention and treatment of DS and in resistive exercises resistance in bariatric subjects.

\section{Methods}

\section{Selection of subjects}

Patients who previously underwent Roux-en-Y gastric bypass surgery, achieved body weight goal (body mass index $<27 \mathrm{~kg} / \mathrm{m}^{2}$ for men and $<25 \mathrm{~kg} / \mathrm{m}^{2}$ for women), had a confirmed diagnosis of DS and were refractory to diet recommendations were initially selected. Inclusion criteria were the complete adherence to an isocaloric (calculated by indirect calorimetry) and high protein (1.5-1.8 g/kg/day) diet plan, proposed resistive exercises (frequency, intensity, and exercise prescription varied among individuals), and age between 18 and 80 years. Patients who lacked regular follow-up were excluded.

\section{Intervention}

Fifty milligrams of acarbose was orally administered 4-5 times a day before meals for 6 months. For those patients who presented with severe flatulence, $120 \mathrm{mg}$ simethicone (b.i.d.) was additionally prescribed. The dose of $50 \mathrm{mg}$ before meals has been extensively studied and standardized, ${ }^{14-16}$ whereas the addition of simethicone was thought to attenuate the most important adverse effects of acarbose use (abdominal cramps, bloating, and flatulence).

\section{Evaluated aspects}

Multiple patterns of DS were evaluated: 1) number of early (0-45 min) DS episodes per week and 2) number of late (45-240 min) DS episodes a week. The DS severity evaluation methods were 1) self-referred intensity of each episode: rated from 1 (almost asymptomatic) to 10 (loss of conscious), 2) Sigstad's scoring system to evaluate diagnosis and intensity of DS (shock +5 ; fainting, syncope, or unconsciousness +4 ; desire to lie down +4 ; dyspnea +3 ; weakness +3 ; sleepiness, apathy +3 ; palpitations +3 ; restlessness +2 ; dizziness +2 ; headaches +1 ; warm, clammy skin, or pallor +1 ; nausea +1 ; abdominal fullness +1 ; borborygmus +1 ; eructation -1 ; vomiting -4 ), and 3) ability to perform resistive exercises: rated from 0 (any effort induces DS symptoms) to 10 (no symptoms, even in intensive weight lifting). Subjects were prospectively instructed to evaluate and write down the frequency and severity of DS episodes and to bring the results at each visit, which happened every 2 weeks for 6 months. We proposed that those patients who presented a significant improvement of DS symptoms could continue the therapy after the end of the study. In each visit, subjects were actively queried about missing doses and irregular use.

\section{Ethical approval}

The proposed study protocol did not provide new or experimental therapies, but analyzed standardized modalities, and therefore approval from an ethics committee was not required. The exemption was issued by the national review board and ethics committee system (Sistema CEP-CONEP - Plataforma 
Brasil), which has formally authorized the intervention without submission to an ethics committee, but required that we provide subjects written informed consent to participate in the study, and containing all the benefits and possible harmful effects of the use of acarbose. We confirm that every patient was fully aware of the purpose of the study and signed informed consent to participate, prior to beginning the use of acarbose. The principles outlined in the Declaration of Helsinki were followed.

\section{Results}

A total of 25 subjects (13 men and 12 women; mean age, 37.2 years) were regularly followed up. None of the patients withdrew or missed doses. Among these, 7 presented with severe flatulence and one patient showed persistent flatulence despite simethicone prescription. None of the subjects stopped acarbose use owing to side effects. Significant improvements were seen in all analyzed parameters: 1) mean number of early DS episodes per week $(2.18-0.31 ; 85.8 \%$ reduction; $p<0.001)$, 2) mean number of late DS episodes per week (2.79-0.12; $95.7 \%$ reduction; $p<0.001), 3$ ) self-referred intensity of each episode (6.1-1.65; 73.0\% reduction; $p<0.001)$, 4) Sigstad's score $(2.36-0.32 ; 86.4 \%$ reduction; $p<0.001)$, and 5$)$ ability to perform resistive exercises $(3.03-7.12 ; 135.0 \%$ improvement; $p<0.001)$. These results are summarized in Table 1. Improvements were observed from the beginning of the use of acarbose and persisted throughout the 6 months of therapy.

Complete remission of DS (except during exercises) was observed in 21 patients ( $84 \%$ of analyzed subjects), which persisted throughout the 6 months of acarbose use in all 21 subjects.

\section{Discussion}

Bariatric surgery reduces metabolic, cardiovascular, and neoplastic risks, overall mortality, and substantially improves the quality of life. However, DS has limited the everyday activities

Table I Summary of the results

\begin{tabular}{llll}
\hline Analyzed parameter & $\begin{array}{l}\text { Before } \\
\text { acarbose }\end{array}$ & $\begin{array}{l}\text { After } \\
\mathbf{6} \text { months } \\
\text { of acarbose }\end{array}$ & $\begin{array}{l}\text { Change, } \% \\
\text { ( } \boldsymbol{p} \text {-value) }\end{array}$ \\
\hline $\begin{array}{l}\text { Mean number of early DS } \\
\text { episodes/week }\end{array}$ & 2.18 & 0.31 & $-85.8(<0.001)$ \\
$\begin{array}{l}\text { Mean number of late DS } \\
\text { episodes/week }\end{array}$ & 2.79 & 0.12 & $-95.7(<0.001)$ \\
$\begin{array}{l}\text { Mean self-referred intensity } \\
\text { of each DS episode }\end{array}$ & 6.1 & 1.65 & $-73.0(<0.01)$ \\
$\begin{array}{l}\text { Sigstad's scoring } \\
\text { Ability to perform resistive } \\
\text { exercises }\end{array}$ & 3.03 & 7.12 & $-86.4(<0.001)$ \\
\hline
\end{tabular}

Abbreviation: DS, dumping syndrome. of several post-bariatric surgery patients and impaired expected improvement in various aspects of quality of living. ${ }^{1,2}$ Little effort has gone into finding effective options to decrease severity and frequency of DS events; moreover, the frequency of severe DS and number of DS-related deaths are likely under reported. Acarbose has been shown to be an inexpensive option that substantially improves overall quality of life of post-bariatric subjects affected by DS, particularly the late dumping. ${ }^{14-16}$

Although early DS is not related to hypoglycemia, the complete understanding of its pathophysiology is still lacking and is assumed to be due to a contraction of the plasma volume as a result of the fluid shifts into the gastrointestinal tract, ${ }^{19}$ which does not fully justify the signs and symptoms observed in early dumping. Possibly, the delay of autonomic and incretin hormones stimulations (once incretins also induce sympathetic and autonomic effects) may at least partly explain the improvement observed in early DS; in fact, increased release of multiple gastrointestinal hormones, including vasoactive agents, incretins, and glucose modulators, in early DS has been described. . $^{40-22}$

To the best of our knowledge, the novelties of this study are that it has been the first to show 1) the important improvement of early DS and complete remission of DS in most patients (84.0\%) who were treated with acarbose and 2) the improvement of exercise capacity among DS subjects who triggered or worsened DS symptoms during physical exertion. Subjective patient testimonies express relevant change in everyday life and the desire to maintain long-term therapy.

Regular intake was observed in all included subjects, probably due to the benefits provided by this drug. Likewise, all patients maintained the use of acarbose after the end of the study, once the improvement observed was significant that it would be an ethical issue to interrupt the proposed therapy. Therefore, we were not able to predict the effects of acarbose discontinuation, although some improvement could be potentially observed after acarbose discontinuation, given the fact that hyperfunction and hypertrophy of the beta-cells, related to rapid glucose absorption in bariatric subjects, are blunted by this drug.

A limitation of the study is that we did not include a control group to study the possible placebo effect of acarbose, although it is unlikely that the observed improvements are due to placebo effect.

\section{Conclusion}

Acarbose induced complete remission of DS in most subjects and substantially decreased the frequency and intensity of both early and late DS events. Further prospective randomized 
placebo-controlled double-blind clinical trials with monitoring of vital signs and glucose should be performed in order to confirm our findings. Until then, acarbose may be treated as an option for refractory DS subjects.

\section{Acknowledgments}

We acknowledge the support of the Corpometria Institute staff team, who helped to manage all the subjects who were included in this study.

\section{Disclosure}

The authors report no conflicts of interest in this work.

\section{References}

1. Ukjela A. Dumping syndrome: pathophysiology and treatment. Nutri Clin Pract. 2005;20(5):517-525.

2. Abell TL, Minocha A. Gastrointestinal complications of bariatric surgery: diagnosis and therapy. Am J Med Sci. 2006;331(4):214-218.

3. Tack J, Arts J, Caenepeel P, De Wulf D, Bisschops R. Pathophysiology, diagnosis and management of postoperative dumping syndrome. Nat Rev Gastroenterol Hepatol. 2009;6(10):583-590.

4. Van Beek AP, Emous M, Laville M, Tack J. Dumping syndrome after esophageal, gastric or bariatric surgery: pathophysiology, diagnosis, and management. Obes Rev. Epub 2016 Oct 17.

5. Lass A. Life after bariatric surgery. Adv Nurse Pract. 2008;16(6):47-48, 50-51.

6. Berg P, McCallum R. Dumping syndrome: a review of the current concepts of pathophysiology, diagnosis, and treatment. Dig Dis Sci. 2016;61(1):11-18.

7. Parrish CR. The clinician's guide to short bowel syndrome. Pract Gastroenterol. 2005;29(9):67.

8. Didden P, Penning C, Masclee AAM. Octreotide therapy in dumping syndrome: analysis of long-term results. Aliment Pharmacol Ther. 2006; 24(9):1367-1375.

9. Li-Ling J, Irving M. Therapeutic value of octreotide for patients with severe dumping syndrome - a review of randomised controlled trials. Postgrad Med J. 2001;77(909):441-442.
10. Oliveira EP, Burini RC. Food-dependent, exercise-induced gastrointestinal distress. J Int Soc Sports Nut.2011;8:12.

11. Livhits M, Mercado C, Yermilov I, et al. Exercise following bariatric surgery: systematic review. Obes Surg. 2010;20(5):657-665.

12. Welch G, Wesolowski C, Piepul B, Kuhn J, Romanelli J, Garb J. Physical activity predicts weight loss following gastric bypass surgery: findings from a support group survey. Obes Surg. 2008;18(5):517-524.

13. Women Fitness Magazine. $2016 \mathrm{Feb}$ print edition. Available from: http:// www.womenfitness.net/dumping_syndrome.htm https://sites.google. $\mathrm{com} / \mathrm{a} / \mathrm{brnamj} 1 . \mathrm{com} /$ health/health-a-z/d/symptoms-causes-risk-factorscomplications-preparing-for-your-appointment-tests-and-diagnosis-treat ments-and-drugs-lifestyle-and-home-remedies-coping-and-support-pre vention-12. Accessed October 29, 2016.

14. De Cunto A, Barbi E, Minen F, Ventura A. Safety and efficacy of highdose acarbose treatment for dumping syndrome. J Pediatr Gastroenterol Nutr. 2011;53(1):113-114.

15. Hasegawa T, Yoneda M, Nakamura K, et al. Long-term effect of alphaglycosidade inhibitor on late dumping syndrome. J Gastroenterol Hepatol. 1998;13(12):1201-1206.

16. Wang C, Pang S, Jiang Q, Duan G, Sun Y, Li M. Treatment with acarbose in severe hypoglycaemia due to late dumping syndrome. West Indian Med J. 2013;62(9):861-863.

17. Ceppa EP, Ceppa DP, Omotosho PA, Dickerson JA 2nd, Park CW, Portenier DD. Algorithm to diagnose etiology of hypoglycemia after Roux-en-Y gastric bypass for morbid obesity: case series and review of the literature. Surg Obes Relat Dis. 2012;8(5):641-647.

18. Mala T. Postprandial hyperinsulinemic hypoglycemia after gastric bypass surgical treatment. Surg Obes Relat Dis. 2014;10(6): $1220-1225$.

19. Elrazek AE, Elbanna AE, Bilasy SE. Medical management of patients after bariatric surgery: principles and guidelines. World J Gastrointest. 2014;6(11):220-228.

20. Tack J. Gastric motor disorders. Best Pract Res Clin Gastroenterol. 2007;21(4):633-644.

21. Mayer EA, Thompson JB, Jehn D, Reedy T, Elashoff J, Meyer JH. Gastric emptying and sieving of solid food and pancreatic and biliary secretion after solid meals in patients with truncal vagotomy and antrectomy. Gastroenterology. 1982;83(1 Pt 2):184-192.

22. Lawaetz O, Blackburn AM, Bloom SR, Aritas Y, Ralphs DN. Gut hormone profile and gastric emptying in the dumping syndrome. A hypothesis concerning the pathogenesis. Scand J Gastroenterol. 1983;18(1):73-80.
Diabetes, Metabolic Syndrome and Obesity: Targets and Therapy is an international, peer-reviewed open-access journal committed to the rapid publication of the latest laboratory and clinical findings in the fields of diabetes, metabolic syndrome and obesity research. Original research, review, case reports, hypothesis formation, expert opinion and commentaries are all considered for publication. The manuscript management system is completely online and includes a very quick and fair peer-review system, which is all easy to use. Visit http://www.dovepress.com/testimonials.php to read real quotes from published authors. 